(C2007 IEEE. Personal use of this material is permitted. However, permission to reprint/republish this material for advertising or promotional purposes or for creating new collective works for resale or redistribution to servers or lists, or to reuse any copyrighted component of this work in other works must be obtained from the IEEE. 


\title{
Variable Digital Filter With Least-Square Criterion and Peak Gain Constraints
}

\author{
Hai Huyen Dam, Antonio Cantoni, Fellow, IEEE, Kok Lay Teo, Senior Member, IEEE, and \\ Sven Nordholm, Senior Member, IEEE
}

\begin{abstract}
Variable digital filters are useful for various signal processing and communication applications where the frequency characteristics, such as fractional delays and cutoff frequencies, can be varied online. In this brief, we present a formulation that allows the tradeoff between the total squared error and the maximum deviation from the desired response in the passband and stopband. With this formulation, the maximum deviation can be reduced below the least-square solution with only a slight change in the performance of the total squared error. Similarly, the total squared error can be reduced below the minmax solution with a minor change in the maximum deviation from the minmax solution.
\end{abstract}

Index Terms-Least-square criterion, min-max criterion, peak gain constraint, variable digital filter (VDF).

\section{INTRODUCTION}

$\mathbf{V}$ ARIABLE digital filters (VDFs) are digital filters with controllable spectral characteristics such as variable cutoff frequency, adjustable passband width and controllable fractional delay [1]-[3]. These spectral characteristics can be varied online. Variable digital filters have many applications in different areas of signal processing and communications. Examples include arbitrary sample rate changers, digital synchronizers and other applications involving online tuning of frequency characteristics [4], [5]. Fractional delay digital filters have various important applications including timing adjustment for digital receivers [4].

The least-square design criterion is commonly used to design Farrow-based [6] finite-impulse response (FIR) VDFs. This criterion gives rise to a quadratic optimization problem [8]. A linear programming technique has also been proposed for the design of variable digital filters with minmax design error criterion [3]. With a minmax criterion, the emphasis is to minimize the maximum amplitude distortion of signals to be passed by a

Manuscript received May 3, 2006. This work was supported in part by ARC under Grant DP0451111 and in part by National ICT Australia (NICTA), through the Australian Government's Backing Australia's Ability initiative, in part through ARC. This paper was recommended by Associate Editor E. A. B. da Silva.

H. H. Dam is with the Western Australian Telecommunications Research Institute and the Department of Mathematics and Statistics, Curtin University of Technology, Bently, WA 6102, Australia (e-mail: dam@watri.org.au).

A. Cantoni is with the Western Australian Telecommunications Research Institute, The University of Western Australia, Nedlands, WA 6907, Australia (e-mail: cantoni@watri.org.au).

K. L. Teo is with the Department of Mathematics and Statistics, Curtin University of Technology, Bently, WA 6102, Australia (e-mail: K.L.Teo@curtin. edu.au).

S. Nordholm is with the Western Australian Telecommunications Research Institute, Curtin University of Technology, Bently, WA 6102, Australia (e-mail: sven@watri.org.au).

Digital Object Identifier 10.1109/TCSII.2006.883102

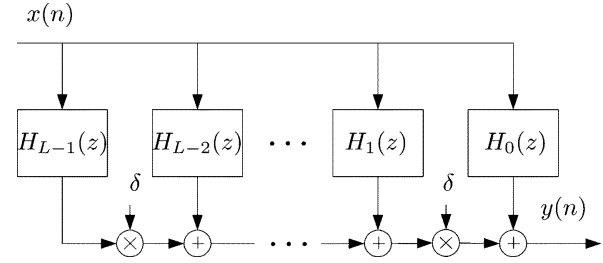

Fig. 1. VDF-Farrow structure.

filter without taking into consideration the error energy. Thus, minmax filters typically have high sidelobe energy. On the other hand, the least-square error criterion is related to the noise gain of the filter. This criterion is simple and easy to formulate. However, it results in the filters with low sidelobe energy but having large errors near the discontinuities in the desired response.

In this brief, we investigate a design formulation for the Farrow structure, [6], which allows a tradeoff between the minmax and least-square criteria. This problem has been initially investigated in [7]. The design formulation can be formulated as a semi-infinite quadratic optimization problem while the minmax problem can be formulated as a semi-infinite linear optimization problem. Two numerical schemes with adaptive grid size are presented for solving the semi-infinite linear and quadratic optimization problems. Following from the general window design [10], we show the tradeoff between the integral squared error and the peak error for VDFs. The minmax and least-square solutions are at the end points of the tradeoff curve. Design examples show that starting from the least-square solution, a reduction in the peak error can be obtained at the expense of a small increase in the integral squared error. Similarly, starting from the minmax solution, a reduction in the integral squared error can be obtained at the expense of a slight increase in the peak error. It is noted that the presented scheme can be applied to the design of bandpass and bandstop filters, proposed in [9].

The brief is organized as follows. The problem formulation and optimization problem is given in Section II. Algorithms for solving linear and quadratic semi-infinite optimization problems that arise are given in Section III. Design examples solved by using the optimization techniques are presented in Section IV. Finally, conclusions are given in Section V.

\section{PROBlem Formulation AND OPTIMIZATION PROBlem}

Consider the design of a VDF [6] depicted in Fig. 1. The structure has $L$ subfilters with $z$-transform $H_{l}(z), 0 \leq l \leq L-1$

$$
H_{l}(z)=\sum_{n=0}^{N-1} h_{l}(n) z^{-n}=\mathbf{h}_{l}^{T} \phi(z)
$$


where $\mathbf{h}_{l}=\left[h_{l}(0), \ldots, h_{l}(N-1)\right]^{T}$ is the impulse response for the $l^{t h}$ subfilter and $\phi(z)=\left[1, \ldots, z^{N-1}\right]$ with $[\cdot]^{T}$ denoting the transpose of a vector $[\cdot]$.

The frequency response of the VDF can be expressed as

$$
H\left(e^{j \omega}, \delta\right)=\sum_{l=0}^{L-1} H_{l}\left(e^{j \omega}\right) \delta^{l}=\mathbf{h}^{T} \mathbf{s}\left(e^{j \omega}, \delta\right)
$$

where $\mathbf{h}=\left[\mathbf{h}_{0}^{T}, \ldots, \mathbf{h}_{L-1}^{T}\right]^{T}$ and

$$
\mathbf{s}\left(e^{j \omega}, \delta\right)=\left[\phi^{T}\left(e^{j \omega}\right) \delta^{0}, \ldots, \phi^{T}\left(e^{j \omega}\right) \delta^{L-1}\right]^{T} .
$$

We consider the design of a VDF with variable cutoff frequency or variable fractional delay. The desired frequency response for the filter can be given as

$$
H_{d}\left(e^{j \omega}, \delta\right)= \begin{cases}e^{-j \omega \tau(\delta)}, & \omega \in \mathcal{P}(\delta) \\ 0, & \omega \in \mathcal{S}(\delta)\end{cases}
$$

where $\mathcal{P}(\delta), \mathcal{S}(\delta)$ and $\tau(\delta)$ are the passband and stopband regions and the desired group delay, respectively, which can depend on the tuning parameter $\delta$. For a low-pass filter, the passband and stopband regions can be given as

$$
\begin{aligned}
& \mathcal{P}(\delta)=\left[-\omega_{p}(\delta), \omega_{p}(\delta)\right] \text { and } \\
& \mathcal{S}(\delta)=\left[-\pi,-\omega_{s}(\delta)\right] \cup\left[\omega_{s}(\delta), \pi\right] .
\end{aligned}
$$

The cutoff frequencies $\omega_{p}(\delta)$ and $\omega_{s}(\delta)$ can be tunable with respect to $\delta$ and in general can be given as

$$
\omega_{p}(\delta)=\omega_{p}+\beta_{p}(\delta) \cdot \delta \text { and } \omega_{s}(\delta)=\omega_{s}+\beta_{s}(\delta) \cdot \delta
$$

where $\omega_{p}$ and $\omega_{s}$ are passband and stopband cutoff frequencies at $\delta=0$. The desired group delay $\tau(\delta)$ is $\tau(\delta)=\tau_{d}+\beta_{\tau}(\delta) \cdot \delta$ where $\tau_{d}$ is the desired group delay for $\delta=0$.

Note that in literature [1], [5], various ranges for $\delta$ have been chosen. In this case, we chose the range of $\delta$ as $\Delta=[0,1]$, [1]. The functions $\beta_{p}(\delta), \beta_{s}(\delta)$ and $\beta_{\tau}(\delta)$ are continuous with respect to the parameter $\delta$.

The filter is optimized over all $\delta \in \Delta$. The integral squared error measure can be defined as the integration of the error deviation between $H\left(e^{j \omega}, \delta\right)$ and the desired frequency response $H_{d}\left(e^{j \omega}, \delta\right)$ over all possibilities of $\delta$ and $\omega$,

$$
E(\mathbf{h})=\int_{\Delta} \int_{\Omega(\delta)} W(\omega, \delta)\left|H\left(e^{j \omega}, \delta\right)-H_{d}\left(e^{j \omega}, \delta\right)\right|^{2} d \omega d \delta
$$

where $\Omega(\delta)=\mathcal{P}(\delta) \cup \mathcal{S}(\delta)$ and $W(\omega, \delta)$ are the weighting function,

$$
W(\omega, \delta)= \begin{cases}W_{p}(\delta), & \text { if } \omega \in \mathcal{P}(\delta) \\ W_{s}(\delta), & \text { if } \omega \in \mathcal{S}(\delta)\end{cases}
$$

with $W_{p}(\delta)$ and $W_{s}(\delta)$ being the positive weighting factors.

Since we consider a real coefficient vector $\mathbf{h}$, the cost function in (3) can be reduced to the following quadratic function:

$$
E(\mathbf{h})=\mathbf{h}^{T} \mathbf{Q h}+\mathbf{p}^{T} \mathbf{h}+c
$$

where $\mathbf{Q}=\mathcal{R}\left\{\int_{\Delta} \int_{\Omega(\delta)} W(\omega, \delta) \mathbf{s}(\omega, \delta) \mathbf{s}^{H}(\omega, \delta) d \omega d \delta\right\}$

$$
\mathbf{p}=-2 \mathcal{R}\left\{\int_{\Delta} \int_{\Omega(\delta)} W(\omega, \delta) \mathbf{s}(\omega, \delta) H_{d}^{*}\left(e^{j \omega}, \delta\right) d \omega d \delta\right\}
$$

and $c=\int_{\Delta} \int_{\Omega(\delta)} W(\omega, \delta)\left|H_{d}\left(e^{j \omega}, \delta\right)\right|^{2} d \omega d \delta$, with [. $]^{H}$ denoting the Hermitian transpose of a complex vector [-].

The least-square solution is obtained by minimizing the quadratic cost function in (4). The solution to this optimization problem can be expressed as $\mathbf{h}_{L S}=-(1 / 2) \mathbf{Q}^{-1} \mathbf{p}$. Denote by $H_{L S}\left(e^{j \omega}, \delta\right)$ the corresponding frequency response of the least-square solution. The maximum deviation level in the passband and stopband for the least-square solution is

$$
\gamma_{L S}=\max _{\delta \in \Delta} \max _{\omega \in \Omega(\delta)} W(\omega, \delta)\left|H_{L S}\left(e^{j \omega}, \delta\right)-H_{d}\left(e^{j \omega}, \delta\right)\right|
$$

with the integral squared error $E_{L S}=E\left(\mathbf{h}_{L S}\right)$.

For the minmax criterion, the optimization problem can be formulated as

$$
\min _{\mathbf{h}} \max _{\delta \in \Delta} \max _{\omega \in \Omega(\delta)} W(\omega, \delta)\left|H\left(e^{j \omega}, \delta\right)-H_{d}\left(e^{j \omega}, \delta\right)\right| .
$$

By introducing a positive parameter $\gamma$, the problem (5) can be re-formulated as

$$
\left\{\begin{array}{l}
\min _{\mathbf{h}} \gamma \\
W(\omega, \delta)\left|H\left(e^{j \omega}, \delta\right)-H_{d}\left(e^{j \omega}, \delta\right)\right| \leq \gamma, \forall \omega \in \Omega(\delta), \delta \in \Delta .
\end{array}\right.
$$

Furthermore, by using the real rotation theorem [11] and letting $\mathbf{z}=\left[\mathbf{h}^{T}, \gamma\right]^{T}$, this problem reduces to the following semiinfinite linear programming problem with respect to the vector $\mathbf{z}$

$$
\left\{\begin{array}{l}
\min _{\mathbf{z}} f(\mathbf{z})=\mathbf{b}^{T} \mathbf{z} \\
g(\omega, \delta, \lambda, \mathbf{z}) \leq 0, \forall \omega \in \Omega(\delta), \delta \in \Delta \text { and } \lambda \in[0,1]
\end{array}\right.
$$

where $\mathbf{b}=\left[\mathbf{0}_{1, N}, 1\right]^{T}$ with $\mathbf{0}_{1, N}$ is a $1 \times N$ zero vector

$$
\begin{aligned}
g(\omega, \delta, \lambda, \mathbf{z}) & =\mathbf{A}(\omega, \delta, \lambda) \mathbf{z}-W(\omega, \delta) \mathcal{R}\left\{H_{d}\left(e^{j \omega}, \delta\right) e^{j 2 \pi \lambda}\right\} \\
\text { and } \mathbf{A}(\omega, \delta, \lambda) & =\left[\left[W(\omega, \delta) \mathcal{R}\left\{\mathbf{s}\left(e^{j \omega}, \delta\right) e^{j 2 \pi \lambda}\right\}\right]^{T},-1\right] .
\end{aligned}
$$

Denote by $\mathbf{z}_{M M}=\left[\mathbf{h}_{M M}^{T}, \gamma_{M M}\right]^{T}$ the optimum solution to the problem (6) with the maximum deviation level $\gamma_{M M}$ and the integral squared error $E_{M M}$. Since the minmax solution has the lowest maximum error deviation while the least-square solution has the lowest integral squared error, we have $E_{L S} \leq$ $E_{M M}$ and $\gamma_{M M} \leq \gamma_{L S}$.

In the following, we present a design criterion that allows the tradeoff between the minmax and the least-square criteria. The optimization problem can be formulated as: minimizing the integral squared error with the maximum error deviation being restricted to be less than or equal to a positive value $\alpha$

$$
W(\omega, \delta)\left|H\left(e^{j \omega}, \delta\right)-H_{d}\left(e^{j \omega}, \delta\right)\right| \leq \alpha, \forall \omega \in \Omega(\delta), \delta \in \Delta
$$

where $\gamma_{M M} \leq \alpha \leq \gamma_{L S}$. Obviously, $\alpha=\gamma_{M M}$ and $\alpha=\gamma_{L S}$ reduce to the minmax and the least-square solutions, respectively. By varying $\alpha$ within the range $\left[\gamma_{M M}, \gamma_{L S}\right]$, we observe the tradeoff between the maximum deviation in the passband, 
stopband, and the integral squared error. The design problem can be formulated as

$$
\left\{\begin{array}{l}
\min _{\mathbf{h}} E(\mathbf{h}) \\
W(\omega, \delta)\left|H\left(e^{j \omega}, \delta\right)-H_{d}\left(e^{j \omega}, \delta\right)\right| \leq \alpha, \forall \omega \in \Omega(\delta), \delta \in \Delta .
\end{array}\right.
$$

By using the real rotation theorem [11], this problem can be reduced to the following semi-infinite quadratic optimization problem

$$
\begin{gathered}
\left\{\begin{array}{l}
\min _{\mathbf{h}} f(\mathbf{h})=\mathbf{h}^{T} \mathbf{Q h}+\mathbf{p}^{T} \mathbf{h}+c \\
g(\omega, \delta, \lambda, \mathbf{h}) \leq \alpha, \forall \omega \in \Omega(\delta), \delta \in \Delta
\end{array}\right. \\
\text { where } g(\omega, \delta, \lambda, \mathbf{h})=\mathbf{A}_{1}(\omega, \delta, \lambda) \mathbf{h}
\end{gathered}
$$

and $\mathbf{A}_{1}(\omega, \delta, \lambda)=\left[W(\omega, \delta) \mathcal{R}\left\{\mathbf{s}\left(e^{j \omega}, \delta\right) e^{j 2 \pi \lambda}\right\}\right]^{T}$.

\section{Algorithms}

The problems (6) and (7) are, respectively, semi-infinite linear and quadratic optimization problems with three continuous parameters $\omega, \delta$ and $\lambda$. In the following, we present the scheme for solving (7).

It can be seen from [11] that if a unit circle generated by $e^{j 2 \pi \lambda}$ for $\lambda \in[0,1]$ is approximated by 16 points, $\hat{\Lambda}=[0,1 / 16, \ldots, 15 / 16]$, then the difference between the absolute complex value and the maximum discretized value is relatively small. Thus, the number of discretization points $K_{\lambda}$ for $\lambda$ is set as $K_{\lambda}=16$. In addition, denote by $\hat{\Delta}$ the discretized set for $\Delta$ with $K_{\delta}$ points. The problem (7) reduces to

$$
\left\{\begin{array}{l}
\min _{\mathbf{h}} f(\mathbf{h}) \\
g(\omega, \delta, \lambda, \mathbf{h}) \leq \alpha, \forall \omega \in \Omega(\delta), \delta \in \hat{\Delta} \text { and } \lambda \in \hat{\Lambda}
\end{array}\right.
$$

If the continuous set $\Omega(\delta)$ for $\omega$ is chosen from a fixed grid set of size $K_{\omega}$ for all $\delta$, then the problem (8) reduces to a quadratic optimization problem with $K_{\omega} K_{\delta} K_{\lambda}$ constraints. For the discretization problem obtained to be a good approximation to the original problem, the integers $K_{\omega}$ and $K_{\delta}$ should be sufficiently large. Thus, the discretization problem has a large number of constraints. To reduce the number of constraints, a near active constraint scheme [12] is used to solve the optimization problem. A discretized optimization method with adaptive schemes for the grid set is employed, where a sequence of adaptive grid points is constructed and the grids are refined gradually.

Let $\mathcal{F}$ denote the feasible set for $(8)$

$$
\mathcal{F}=\{\mathbf{h}: g(\omega, \delta, \lambda, \mathbf{h}) \leq \alpha, \forall \omega \in \Omega(\delta), \delta \in \hat{\Delta} \text { and } \lambda \in \hat{\Lambda}\}
$$

We have the following properties.

1) Property 3.1: There exists an $M \geq 0$ such that for any $\mathbf{h}$ bounded in $\mathcal{F}, \delta \in \hat{\Delta}$ and $\omega, \omega_{1} \in \mathcal{P}(\bar{\delta})$ or $\mathcal{S}(\delta), \lambda \in \hat{\Lambda}$, we have

$$
\left|g(\omega, \delta, \lambda, \mathbf{h})-g\left(\omega_{1}, \delta, \lambda, \mathbf{h}\right)\right| \leq M\left|\omega-\omega_{1}\right|
$$

Proof: Assume that $\omega$ and $\omega_{1} \in \mathcal{P}(\delta)$, which is the passband. We have

$$
\begin{aligned}
\mid g(\omega, \delta, \lambda, \mathbf{h}) & -g\left(\omega_{1}, \delta, \lambda, \mathbf{h}\right) \mid \\
\leq & W_{p}(\delta)\left|\mathcal{R}\left\{\left(\mathbf{s}\left(e^{j \omega}, \delta\right)-\mathbf{s}\left(e^{j \omega_{1}}, \delta\right)\right) e^{j 2 \pi \lambda}\right\}^{T} \mathbf{h}\right| \\
& +W_{p}(\delta)\left|\left(e^{j \omega \tau(\delta)}-e^{j \omega_{1} \tau(\delta)}\right) e^{j 2 \pi \lambda}\right| \\
\leq & W_{p}(\delta) \sum_{l, n}\left|\left(e^{j \omega n}-e^{j \omega_{1} n}\right) \delta^{l}\right| \cdot\left|\max _{l, n}\right| h_{l}(n) \mid \\
& +W_{p}(\delta)\left|e^{j \omega \tau(\delta)}-e^{j \omega_{1} \tau(\delta)}\right| .
\end{aligned}
$$

Since

$$
\begin{aligned}
\left|e^{j \omega n}-e^{j \omega_{1} n}\right| & \\
& =\left|e^{j \omega_{1} n}\left(e^{j\left(\omega-\omega_{1}\right) n}-1\right)\right| \\
& =\sqrt{\sin ^{2}\left(\left(\omega-\omega_{1}\right) n\right)+\left(\cos \left(\left(\omega-\omega_{1}\right) n\right)-1\right)^{2}} \\
& =\sqrt{\sin ^{2}\left(\left(\omega-\omega_{1}\right) n\right)+\left(2 \sin ^{2}\left(\frac{n\left(\omega-\omega_{1}\right)}{2}\right)\right)^{2}} \\
& \leq \sqrt{\left(\left(\omega-\omega_{1}\right) n\right)^{2}+\left(\frac{2\left(\omega-\omega_{1}\right) n}{2}\right)^{2}}=\sqrt{2} n\left|\omega-\omega_{1}\right|
\end{aligned}
$$

and $\mathbf{h}$ is bounded, we have (10) where $M=M_{p}$ in the passband with

$$
M_{p}=\max _{\delta} \sqrt{2} W_{p}(\delta)\left(\sup _{l, n}\left|h_{l}(n)\right| \sum_{l, n} n\left|\delta^{l}\right|+|\tau(\delta)|\right) .
$$

Similarly, it can be shown that there exists $M_{s}$ so that (10) is satisfied in the stopband, i.e., for all $\omega$ and $\omega_{1} \in \mathcal{S}(\delta)$. Thus, $M$ is chosen $M=\max \left\{M_{p}, M_{s}\right\}$.

2) Property 3.2: For all $\epsilon>0$ there exists a grid $\Omega_{d}(\delta) \subset$ $\Omega(\delta), \delta \in \hat{\Delta}$, such that

$$
\max _{\delta \in \hat{\Delta}} \max _{\omega \in \Omega(\delta)} \min _{\omega_{1} \in \Omega_{d}(\delta)}\left|\omega-\omega_{1}\right| \leq \frac{\epsilon}{M}
$$

Any feasible solution to

$$
\left\{\begin{array}{l}
\min _{\mathbf{h}} f(\mathbf{h}) \\
g(\omega, \delta, \lambda, \mathbf{h}) \leq \alpha-\epsilon, \forall \omega \in \Omega_{d}(\delta), \delta \in \hat{\Delta} \text { and } \lambda \in \hat{\Lambda}
\end{array}\right.
$$

is also a feasible solution to (8).

Proof: Let $\mathbf{h}$ be a feasible solution of (14). For all $\delta \in \hat{\Delta}$ and $\omega \in \Omega(\delta)$, it follows from (13) that there exist an $\omega_{1} \in$ $\Omega_{d}(\delta)$ in the same region as $\omega$, such that $\left|\omega-\omega_{1}\right| \leq \epsilon / M$. Thus

$$
\begin{aligned}
g(\omega, \delta, \lambda, \mathbf{h})-g\left(\omega_{1}, \delta, \lambda, \mathbf{h}\right) & \leq\left|g(\omega, \delta, \lambda, \mathbf{h})-g\left(\omega_{1}, \delta, \lambda, \mathbf{h}\right)\right| \\
& \leq M\left|\omega-\omega_{1}\right| \leq \epsilon .
\end{aligned}
$$

Hence, $g(\omega, \delta, \lambda, \mathbf{h}) \leq \epsilon+g\left(\omega_{1}, \delta, \lambda, \mathbf{h}\right) \leq \alpha$. This completes the proof.

In many cases, the number of constraints turns out to be too large to be handled. Thus, a near active set constraint scheme is used which includes a procedure to eliminate unnecessary 
constraints in (14), [12]. This can be done by restricting $\omega$ to a smaller subset $\Omega_{d}^{\epsilon_{1}}(\delta) \subset \Omega_{d}(\delta)$

$$
\Omega_{d}^{\epsilon_{1}}(\delta)=\left\{\omega \in \Omega_{d}(\delta): g(\omega, \delta, \lambda, \mathbf{h}) \geq \alpha-\epsilon_{1} \text { for some } \lambda\right\}
$$

where $\epsilon_{1}>\epsilon$. The problem (14) with the smaller subset $\Omega_{d}^{\epsilon_{1}}(\delta)$ becomes

$$
\left\{\begin{array}{l}
\min _{\mathbf{z}} f(\mathbf{z}) \\
g(\omega, \delta, \lambda, \mathbf{h}) \leq-\epsilon, \forall \omega \in \Omega_{d}^{\epsilon_{1}}(\delta), \delta \in \hat{\Delta} \text { and } \lambda \in \hat{\Lambda} .
\end{array}\right.
$$

The advantage of the method is that it concentrates on the constraints near the active points. In general, the number of points in $\Omega_{d}^{\epsilon_{1}}(\delta)$ would be significantly smaller than the number of points in $\Omega_{d}(\delta)$. Thus, the complexity for solving (15) is considerably less than solving (14).

The adaptive discretization scheme for the problem (14) is presented in the following.

3) Procedure 3.1: Discretized quadratic programming problem using adaptive grid scheme.

- Step 1: Set the number of discretization points $K_{\delta}$ for $\Delta$ with the discretization set $\hat{\Delta}$. Initialize two positive numbers $\epsilon$ and $\epsilon_{1}$ where $\epsilon_{1}>\epsilon$. In this brief, the value of $\epsilon_{1}$ is chosen as $\epsilon_{1}=\max \left\{2 \epsilon, 10^{-2}\right.$. $\}$ Set the value of $K_{\omega}$ depending on $\epsilon$. Denote by $\hat{\Omega}(\delta)$ the corresponding uniform discretization set for $\Omega(\delta)$ with $K_{\omega}$ grid points.

- Step 2: If $\mathbf{h}$ has not been initialized, then set $\mathcal{X}$ as the set consisting of all discretized $(\omega, \delta)$ in Step 1 and go to Step 3. Otherwise, let $\mathcal{X}$ be the set consisting of $(\omega, \delta)$ such that

$$
\begin{aligned}
\mathcal{X}=\{(\omega, \delta), \omega \in \hat{\Omega}(\delta), \delta \in \hat{\Delta}: W(\omega, \delta) \\
\left.\cdot\left|\mathbf{h}^{T} \mathbf{s}\left(e^{j \omega}, \delta\right)-H_{d}\left(e^{j \omega}, \delta\right)\right| \geq \alpha-\epsilon_{1}\right\} .
\end{aligned}
$$

As can be seen from (16), we do not need to discretize $\lambda$.

- Step 3: Solve the problem (8) with the constraint $g(\omega, \delta, \lambda, \mathbf{h}) \leq \alpha-\epsilon$ where $(\omega, \delta) \in \mathcal{X}, \lambda \in \hat{\Lambda}$ and $\mathbf{h}$ as the initial solution. Update $\mathbf{h}$ as the solution obtained.

- Step 4: If $K_{\omega} \leq K_{\omega}^{\max }$ where $K_{\omega}^{\max }$ is the maximum number of discretized points for $\omega$, then set $2 K_{\omega} \rightarrow K_{\omega}$ and update the uniform discretization sets $\hat{\Omega}(\delta)$ for all $\delta \in \hat{\Delta}$. Otherwise, $\hat{\Omega}(\delta)$ remains the same. If $\epsilon$ is greater than a small tolerance, then reduce the value of $\epsilon, \epsilon / 2 \rightarrow \epsilon$, update the value of $\epsilon_{1}$ and return to Step 2. Otherwise, go to Step 5.

- Step 5: Stop the procedure.

For the problem (6), a scheme similar to that described in Procedure 3.1 can be used for the discretized linear optimization problem with adaptive grid points. The variable in this case is $\mathbf{z}=\left[\mathbf{h}^{T}, \gamma\right]^{T}$ instead of $\mathbf{h}$.

\section{Design EXAmples}

Case 1: Consider the design of a low-pass VDF with variable cutoff frequency. The desired delay $\tau(\omega)$ is constant with respect to the parameter $\delta$. The passband and stopband cutoff frequencies $\omega_{p}(\delta)$ and $\omega_{s}(\delta)$ change linearly with respect to $\delta$ with $\beta_{p}(\delta)=\beta_{s}(\delta)=0.2 \delta \pi$ for all $\delta \in[0,1]$.

Initial passband and stopband cutoff frequencies are $\omega_{p}=0.2 \pi$ and $\omega_{s}=0.4 \pi$. Thus, when $\delta$ changes from 0 to 1 ,

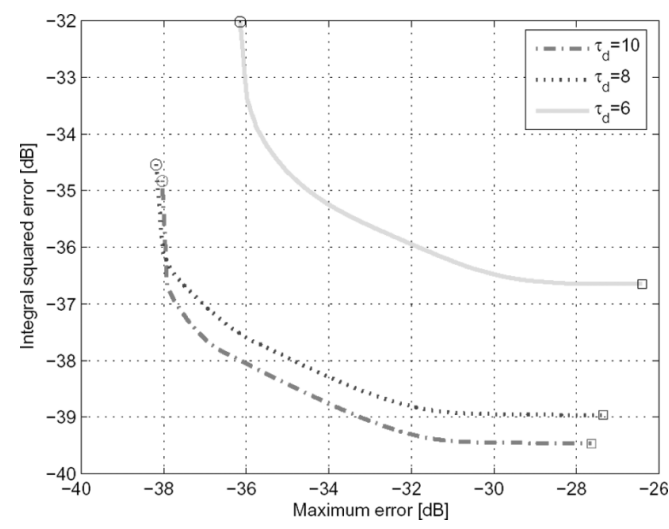

Fig. 2. VDF with variable cutoff frequency. Integral squared error versus minmax error for different desired delay $\tau_{d}$.

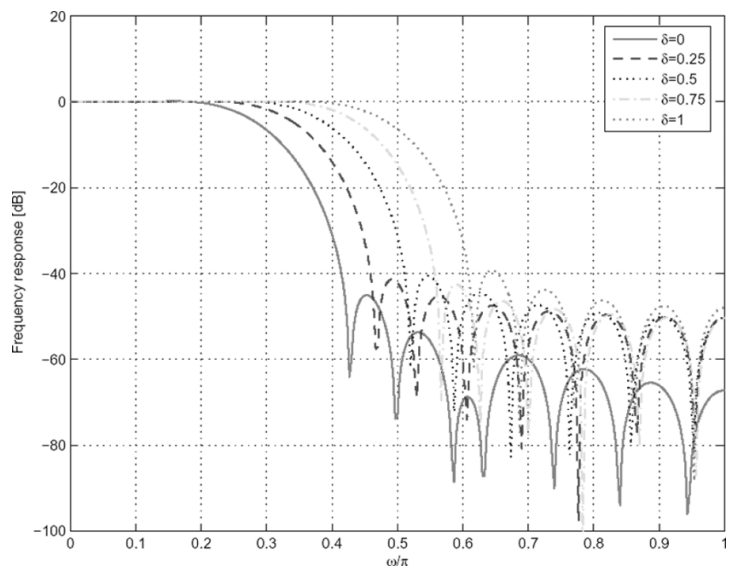

Fig. 3. VDF with variable cutoff frequency. Frequency response of the filters with least-square criterion and $\tau(\delta)=\tau_{d}=10$.

the normalized passband region increases from $[-0.2 \pi, 0.2 \pi]$ to $[-0.4 \pi, 0.4 \pi]$ while the stopband region reduces from $[-\pi,-0.4 \pi] \cup[0.4 \pi, \pi]$ to $[-\pi,-0.6 \pi] \cup[0.6 \pi, \pi]$. The length of the filter is $N=21$ and $L=5$. The weighting function is one in the passband and the stopband, $W_{p}=W_{s}=1$. In addition, the value of $K_{\delta}$ is $K_{\delta}=128$ while $K_{\omega}^{\max }=256$.

Fig. 2 shows the tradeoff between the integral squared error and the maximum error for cases with the desired delay being reduced from 10 to 6 . The least-square and minmax solutions are the extreme points of the curves. It can be seen that the maximum error can be reduced from the least-square solution with a minor change in the integral squared error by incorporating the constraints in the maximum deviation. Similarly, the integral squared error can be reduced from the minmax solution with a minor change in the maximum error. In addition, the maximum error and the integral squared error increase with a reduction in the desired delay.

Figs. 3 and 4 show the frequency responses of the VDF with least-square and minmax criteria. The passband cutoff frequency increases from $0.2 \pi$ to $0.4 \pi$. The minmax filters have approximately the same levels in the stopband while the least-square filters have low stopband energy.

Case 2: Consider the design of a low-pass VDF over continuous tuning parameter $\delta$ with variable delay. The passband and stopband regions are kept constant with $\beta_{p}(\delta)=\beta_{s}(\delta)=0, \forall \delta$. The passband and stopband cutoff frequencies are given by $\omega_{p}(\delta)=\omega_{p}=0.2 \pi$ and $\omega_{s}(\delta)=\omega_{s}=0.4 \pi, \forall \delta$. The desired delay changes linearly over one sample delay with $\beta_{\tau}(\delta)=\delta$. Thus, when $\delta=0$, we 


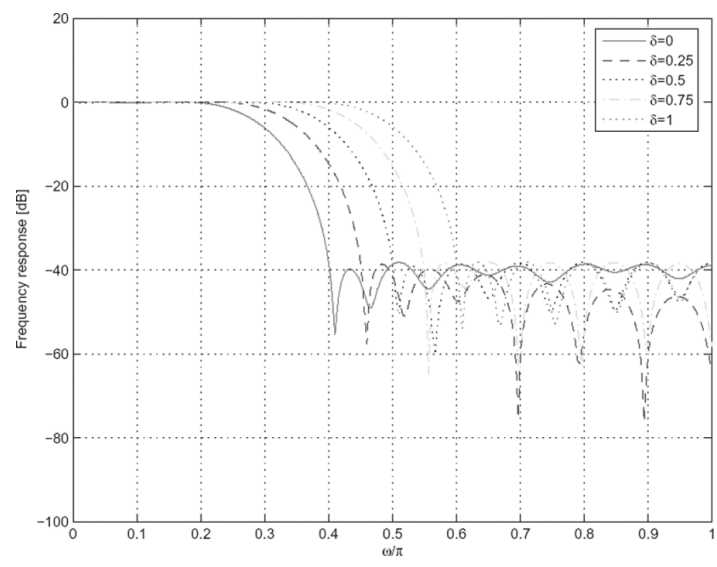

Fig. 4. VDF with variable cutoff frequency. Frequency response of of VDFs with minmax criterion and $\tau(\delta)=\tau_{d}=10$.

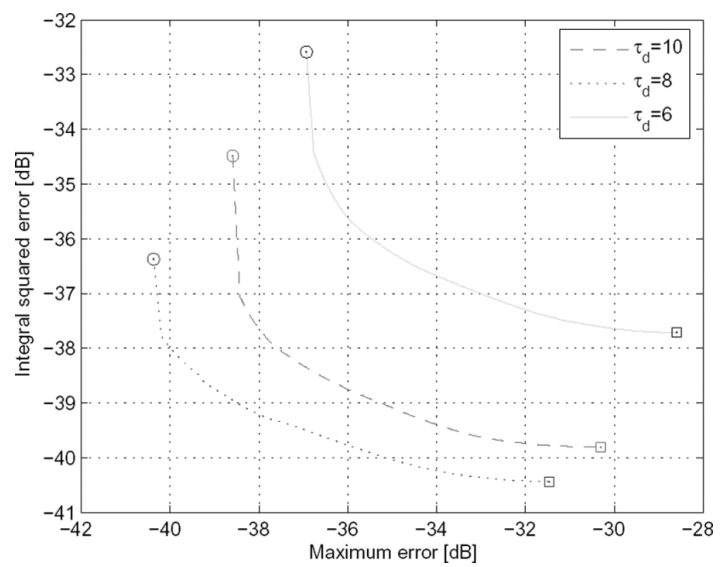

Fig. 5. VDF with variable delay. Integral squared error versus maximum error for different $\tau_{d}$.

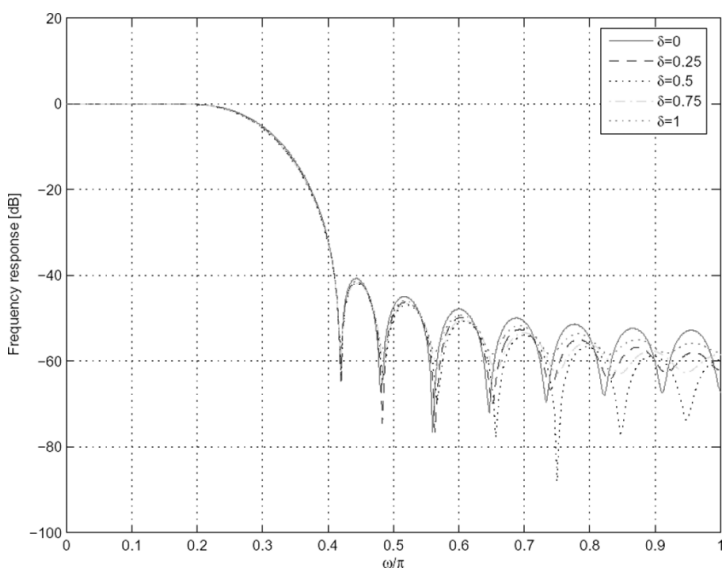

Fig. 6. VDF with variable delay. Frequency response of the filters with leastsquare criterion and $\tau_{d}=6$ for different $\delta$.

have $\tau(\delta)=\tau_{d}$ and when $\delta=1$ we have $\tau(\delta)=\tau_{d}+1$. The values of $N$ and $L$ are chosen the same as in Example 1.

The tradeoff between the least-square and the minmax solutions is shown in Fig. 5 for cases with the desired delay reduced from 10 to 6 . The maximum error and integral squared error are increased when the desired delay is decreased. Similar to the first case, the maximum error can be reduced from the least-square solution with a minor change on the integral squared error while the integral squared error can be reduced from the minmax solution.

Figs. 6 and 7 plot the frequency response and the group delay for the optimized VDF over continuous tuning parameter range

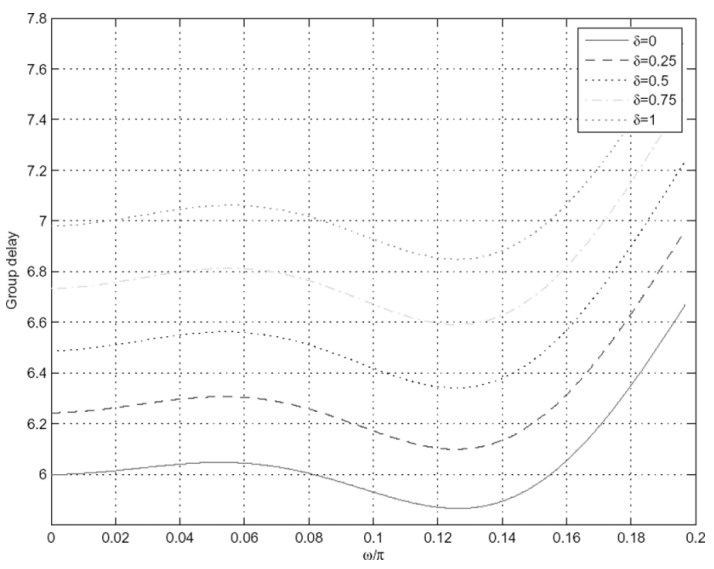

Fig. 7. Group delay VDF with variable delay and least-squared criterion with $\tau_{d}=6$ and different $\delta$.

$\delta$ with the least-square criterion. The desired group delay is increased from $\tau_{d}=6$ to $\tau_{d}+1=7$.

\section{CONCLUSION}

In this brief, we have investigated the design of VDF with least-square criterion and peak gain constraint. We have shown that a tradeoff can be achieved between the maximum error and the total squared error. The maximum deviation can be reduced below the least-square solution with a minor change in the performance of the total squared error. Similarly, the total squared error can be considerably reduced below the minmax solution with a slightly increase in the maximum deviation from the minmax solution. In addition, efficient numerical schemes with adaptive grid size are presented for solving the optimization problems.

\section{REFERENCES}

[1] C. K. S. Pun, S. C. Chan, K. S. Yeung, and K. L. Ho, "On the design and implementation of FIR and IIR digital filters with variable frequency charactericstics," IEEE Trans. Circuits Syst. II, Analog Digit. Signal Process., vol. 49, no. 11, pp. 689-703, Nov. 2002.

[2] H. Zhao and J. Yu, "A simple and efficient design of variable fractional delay FIR filter," IEEE Trans.Circuits Syst. II, Exp. Briefs, vol. 53, no. 2, pp. 157-160, Feb. 2006.

[3] H. Johansson and P. Lowenborg, "On the design of adjustable fractional delay FIR filters," IEEE Trans. Circuits Syst. II, Analog Digit. Signal Process., vol. 50, no. 4, pp. 164-169, Apr. 2003.

[4] T. I. Laakso, V. Valimaki, M. Karjalainen, and U. K. Laine, "Splitting the unit delay: Tools for fractional delay filter design," IEEE Signal Process. Mag., vol. 13, no. 1, pp. 30-60, Jan. 1996.

[5] K. Khamei, A. Nabavi, and S. Hessabi, "Design of variable fractional delay FIR filters using genetic algorithm," in Proc. IEEE Int. Conf. Electronics, Circuits Systems, Dec. 2003, vol. 1, pp. 48-51.

[6] C. W. Farrow, "A continuously variable digital delay element," in Proc. IEEE Int. Symp. Circuits Syst., Jun. 1988, vol. 3, pp. 2641-2645.

[7] H. H. Dam, A. Cantoni, K. L. Teo, and S. Nordholm, "Variable digital filter design with least-square criterion subject to peak gain constraints," in CD-ROM. Eur. Signal Process. Conf., Sep. 2006.

[8] T. B. Deng, "Discretization-free design of variable fractional-delay FIR digital filters," IEEE Trans.Circuits Syst. II, Analog Digit. Signal Process., vol. 48, no. 6, pp. 637-644, Jun. 2001.

[9] P. Lowenborg and H. Johansson, "Minmax design of adjustable-bandwidth linear-phase FIR filters," IEEE Trans. Circuits Syst. I, Reg. Papers, vol. 53, no. 2, pp. 431-439, Feb. 2006.

[10] J. W. Adams, "FIR digital filters with least-squares stopbands subject to peak-gain cosntraints," IEEE Trans. Circuits Syst., vol. 39, no. 4, pp. 376-388, Apr. 1991.

[11] T. W. Parks and C. S. Burrus, Digital Filter Design. New York: Wiley, 1987.

[12] K. L. Teo, X. Q. Yang, and L. S. Jennings, "Computational discretization algorithms for functional inequality constrained optimization," $A n$ nuals Oper. Res., pp. 215-234, 2000. 